\title{
Corrigendum: Bazi Bushen Capsule Alleviates Post-Menopausal Atherosclerosis via GPER1-Dependent Anti-Inflammatory and Anti-Apoptotic Effects
}

\author{
Dan Huang ${ }^{1,2 \dagger}$, Xindong Wang ${ }^{1,2 \dagger}$, Yunhong Zhu ${ }^{1,2}$, Juexiao Gong ${ }^{1,2}$, Junqing Liang ${ }^{3}$, \\ Yanfei Song ${ }^{3}$, Yiyan Zhang ${ }^{1,2}$, Linsheng Liu $^{4 *}$ and Cong Wei ${ }^{3 *}$ \\ ${ }^{1}$ Affiliated Hospital of Integrated Traditional Chinese and Western Medicine, Nanjing University of Chinese Medicine, Nanjing \\ China, ${ }^{2}$ Jiangsu Province Academy of Traditional Chinese Medicine, Nanjing, China, ${ }^{3}$ National Key Laboratory of Collateral \\ Disease Research and Innovative Chinese Medicine, Shijiazhuang, China, ${ }^{4}$ Department of Clinical Pharmacology, The First \\ Affiliated Hospital of Soochow University, Suzhou, China
}

Keywords: atherosclerosis, bazi bushen capsule, lipid profile, inflammation, GPER1 antagonist, apoptosis

\section{OPEN ACCESS}

Edited and reviewed by: Hongzhi Du, Hubei University of Chinese Medicine,

China

*Correspondence: Linsheng Liu linsheng_liu@126.com

Cong Wei weicong@yiling.cn

${ }^{+}$These authors have contributed equally to this work

Specialty section: This article was submitted to Ethnopharmacology,

a section of the journal

Frontiers in Pharmacology

Received: 13 September 2021 Accepted: 17 September 2021

Published: 04 October 2021

Citation:

Huang D, Wang $X$, Zhu Y, Gong J, Liang J, Song $Y$, Zhang $Y$, Liu L and Wei C (2021) Corrigendum: Bazi Bushen Capsule Alleviates Post-

Menopausal Atherosclerosis via GPER1-Dependent Anti-Inflammatory and Anti-Apoptotic Effects. Front. Pharmacol. 12:774792. doi: 10.3389/fphar.2021.774792

\section{A Corrigendum on}

Bazi Bushen Capsule Alleviates Post-Menopausal Atherosclerosis via GPER1-Dependent AntiInflammatory and Anti-Apoptotic Effects

by Huang, D., Wang, X., Zhu, Y., Gong, J., Liang, J., Song, Y., Zhang, Y., Liu, L., and Wei, C. (2021). Bazi Bushen Capsule Alleviates Post-Menopausal Atherosclerosis via GPER1-Dependent AntiInflammatory and Anti-Apoptotic Effects. Front. Pharmacol. 12:658,998. doi:10.3389/fphar.2021. 658998

In the original article, there was a mistake in Table $\mathbf{1}$ as published. BZBS is a post-marketing drug, and there is an error in the components of BZBS and ratio amounts in application (Li et al., 2021). The corrected Table 1 appears below.

The authors apologize for this error and state that this does not change the scientific conclusions of the article in any way. The original article has been updated.

\section{REFERENCE}

Li, L., Chen, B., An, T., Zhang, H., Xia, B., Li, R., et al. (2021). BaZiBuShen Alleviates Altered Testicular Morphology and Spermatogenesis and Modulates Sirt6/P53 and Sirt6/NF-Kb Pathways in Aging Mice Induced by D-Galactose and NaNO2. J. Ethnopharmacol 271, 113810. doi:10.1016/j.jep.2021.113810

Publisher's Note: All claims expressed in this article are solely those of the authors and do not necessarily represent those of their affiliated organizations, or those of the publisher, the editors and the reviewers. Any product that may be evaluated in this article, or claim that may be made by its manufacturer, is not guaranteed or endorsed by the publisher.

Copyright $\odot 2021$ Huang, Wang, Zhu, Gong, Liang, Song, Zhang, Liu and Wei. This is an open-access article distributed under the terms of the Creative Commons Attribution License (CC BY). The use, distribution or reproduction in other forums is permitted, provided the original author(s) and the copyright owner(s) are credited and that the original publication in this journal is cited, in accordance with accepted academic practice. No use, distribution or reproduction is permitted which does not comply with these terms. 
TABLE 1 | The ratio of the plants present in the preparation of BZBS.

Plants

Amount

in application (ratio)

Cuscuta chinensis Lam. [Convolvulaceae; Cuscutae semen]

Lycium barbarum L. [Solanaceae; Lycii cortex]

Schisandra chinensis (Turcz.) Baill. [Schisandraceae; Schisandrae chinensis fructus]

Cnidium monnieri (L.) Cusson [Apiaceae; Cnidii fructus]

Rosa laevigata Michx. [Rosaceae; Rosae laevigatae fructus]

Rubus chingii Hu [Rosaceae; Rubi fructus]

Allium tuberosum Rottler ex Spreng. [Amaryllidaceae; Allii tuberosi semen]

Toosendan fructus [Meliaceae; Fuctus Toosendan]

Epimedium brevicornu Maxim. [ Berberidaceae; Epimedii folium]

Morindae officinalis radix [Rubiaceae; Radix Morindae Offcinalis]

Cistanche deserticola Ma [Orobanchaceae; Cistanches herba]

Rehmannia root [Orobanchaceae; Radix Rehmanniae Recens]

Cyathula officinalis K.C.Kuan [Amaranthaceae; Cyathulae radix]

Panax ginseng C.A.Mey. [Araliaceae; Ginseng radix et rhizoma]

Cervus nippon Temminck [Cervidae; Cervi Cornu Pantotrichum]

Hippocampus Kelloggi [Syngnathidae; Hippocampus] 